\title{
La Fontaine et la tradition européenne de la fable chez l'auteur mexicain Fernández de Lizardi
}

\author{
Strosetzki, Christoph
}

First published in:

La Fontaine et la tradition européenne de la fable : actes du colloque de Chateau-Thierry (16-18 juin 1989). In: Le fablier : revue des Amis de Jean de La Fontaine, No 2: La fable dans le monde moderne et contemporain, 1990, p. 43-49

ISSN: 0996-6560 


\title{
LA FONTAINE ET LA TRADITION EUROPÉENNE DE LA FABLE CHEZ L'AUTEUR MEXICAIN FERNÁNDEZ DE LIZARDI
}

\author{
par Christoph STROSETZKI
}

En transposant ses fables - qui sont en partie la peinture de la société française de la seconde moitié du XVII ${ }^{\mathrm{e}}$ siècle - dans le monde animal, La Fontaine peut se permettre de critiquer, indirectement, non seulement les incommodités et les faiblesses de cette société, mais aussi les discordes qui naissent de l'affrontement des diverses coteries ${ }^{(1)}$, sans que ni lui ni son œuvre ne courussent le danger d'être réprimés par un État dont le pouvoir absolu était incontestable. En considération de ce qui était arrivé à Fouquet, son protecteur, la prudence était donc pour La Fontaine de nécessité première.

Le souci de circonspection était aussi l'une des préoccupations majeures des auteurs contemporains de Lizardi. En effet, alors que l'Espagne était, en 1808 , occupée par les troupes napoléoniennes, on voyait surgir au Mexique une population voulant se libérer du joug de la puissance coloniale qu'exerçait la péninsule ibérique. Des émeutes et conflits opposaient les Espagnols et leurs sympathisants aux Créoles issus de la classe moyenne. Lorsqu'en 1813, le vice-roi Venegas proclama au Mexique la constitution libérale espagnole de Cadix, il y eut en outre de nombreuses confrontations entre traditionnalistes et libéraux. C'est dans ce contexte politique que José Joaquín Fernández de Lizardi publia en 1817 ses fables.

Lizardi vécut de 1776 à 1827 au Mexique. Après avoir achevé ses études au Colegio de San Ildefonso et s'être adonné à des activités juridiques, il se consacra à la littérature. Il publia tout d'abord la revue libérale El pensador mexicano. Ce titre, qui était tout un programme, devint son pseudonyme. Lorsque la liberté de la presse fut supprimée, c'està-dire en 1812 , il se vit condamné à sept mois de prison. Désormais plus prudent, il dissimula ses thèses, qu'il illustre aussi parfois à travers le théâtre ${ }^{(2)}$, en partie sous une forme littéraire. C'est ainsi qu'il écrivit, en 1816, le premier roman picaresque latinoaméricain, El Periquillo Sarniento, dans lequel la réflexion domine sur les composantes narratives ${ }^{(3)}$. Les fables de Lizardi seraient-elles donc, à l'aide de moyens littéraires, tout différents, la prolongation des activités journalistiques de l'auteur? C'est la question que nous allons nous poser dans cet article.

Les fables étaient déjà présentes dans les travaux de journaliste de Lizardi. Ainsi fait-il précéder, en 1812 , le premier numéro de sa revue El pensador mexicano d'une épigraphe qui est tirée du prologue que le fabuliste Ésope composa pour son troisième livre $^{(4)}$. Quand il fait parler, dans un dialogue publié dans sa revue, le taureau avec le cheval, il indique bien que, tout comme Ésope, Phèdre, Iriarte et Samaniego, il possède le don de comprendre le langage animal ${ }^{(5)}$. Il utilise à plusieurs reprises les fables dans sa revue, en outre, comme moyen d'expres$\operatorname{sion}^{(6)}$. Ainsi entame-t-il en 1813 le numéro 10 de son périodique par la fable de la discorde entre le lapin et le lévrier. Dans la morale de cette fable,

(1) CF. J. Grimm, "La représentation de la société de cour dans Les Fables de La Fontaine", in : Lirtératures classiques 11, 1989: cf. aussi Jürgen Grimm, La Fontaines Fabeln, Darmstadt 1976, p. 30f, 91; cf. surtout pour le XVIII" siècle quant à sá conception de la fable et sa stylisation de La Fontaine qui devient le martyr osant formuler, à l'encontre du roi, des jugements défavorables : ibidem, p. 41 et suiv.; cl. aussi Pierre Boutang, La Fontaine politique, Paris 1981

(2) Dans le monologue Arcabuceado il commente les conséquences négatives qui dćcoulent chez les Mexicains du inanque d'éducation. Dans Don Agustín de Irurbide il illustre le fait que c'est la flatteric qui induit les hommes d'État en crrcur. Dans El negro sensible il s'érige contre l'esclavage et dans $L a$ lragedia del padre Arenas contrc la corruption dans le domaine politique. Cf. José Joaquín Fernández de Lizardi, Obras II, Teatro, Jacobo Chencinscy (ed.), Mexico 1965 (Univ. Nacional Aut. de México)

(3) Cf. Dieter Janik, "El Periquillo Samiento de J. J. Fernández de Lizardi : una normativa vacilantc (sociedad-naturaleza y religión-razón)", in : Ibero-Amerikanisches Archiv N.F., 13 , H.1, 1987, p. 49 et suiv.; Sergio Fernández, "El mensaje del Periquillo en el momento de la independancia", in : Filosofia y latras, 47-48, 1952, P. 275-286.

(4) Dans sa propre traduction libre qui suit le texte en latin il écrit : "No es mi intención señalar en particular a ninguno, sino sólo manifestar los vicios y ridiculeces de los hombres. Vete, pues, de aquí, envidia, no ladre tu malicia sin motivo." José Joaquín Fernández de Lizardi, Obras III, EI Pensador. Mexicano, Jacobo Chencinscy (ed.), Mexico 1965 (Univ. Nacional Aut. de México), p. 33.

(5) Fernández de Lizardi, El Pensador Mexicano, p. 477.

(6) Cr. aussi Fcrnández de Lizardi, El Pensador Mexicano, p. $297,493,507$. 
l'auteur nous indique que le plus faible ne doit pas entrer en lutte manifeste contre le plus fort, mais plutôt agir avec prudence. Ce qui correspond bien aux propres problèmes de Lizardi, que le journaliste expose à ses lecteurs en faisant référence à la fable : "Yo siento el haber de dar gusto a algunas personas que me importunan a ratos lo bastante para que diga lo que ellas o no saben, o no quieren, o no pueden decir, de modo que pretenden que sea yo por ahora el órgano de la opinión común" (7).

Une comparaison entre les fables de Lizardi et celles de La Fontaine nous permet de prendre, comme point de départ soit la constellation des figures qui entrent en scène, ou bien des objets, soit la leçon que chacune de ces fables nous transmet. En tenant compte de l'énorme influence de la culture française sur la culture mexicaine du XIX ${ }^{\mathrm{c}}$ siècle ${ }^{(8)}$, et du prestige dont jouissait la littérature française à l'époque du Roi Soleil, pour Lizardi ${ }^{(9)}$, il est normal de se poser la question de l'influence qu'exerça La Fontaine sur Lizardi, sans pour cela contester l'importance, pour ce dernier, des auteurs français du siècle des lumières ${ }^{(10)}$ ou d'auteurs espagnols comme Iriarte ${ }^{(11)}$ et Samaniego ${ }^{(12)}$, d'autant plus que ceux-ci ont aussi subi l'influence du célèbre fabuliste français ${ }^{(13)}$. C'est justement le rôle important réservé aux composantes didactiques qui font la caractéristique des fables au XVIII ${ }^{\mathrm{c}}$ siècle $^{(14)}$. Nous aimerions donc faire l'analyse des fables de Lizardi en tenant compte, en arrière-plan, de ses travaux journalistiques.

La première fable de Lizardi, celle qui suit le prologue, a pour titre Los Lisiados al espejo, y el autor. L'auteur y dépeint le comportement exaspéré de deux frères à qui il tend un miroir grâce auquel ceux-ci peuvent percevoir leurs vices. L'auteur leur fait ensuite comprendre que ce n'est pas le miroir qui est le responsable de leur reflet mais bien euxmêmes ${ }^{(15)}$. Dans les deux derniers vers il se réfère à ses propres lecteurs :

\section{¡Ojalá entiendan el cuento \\ algunos de mis lectores!}

De la même façon, La Fontaine se réfère à la fonction instructive de la littérature, lorsque, dans la fable L'Homme et son image, il met en scène un personnage dont la perfection et l'assurance reposent sur le fait qu'il évite à tout prix la rencontre avec son reflet dans le miroir. Ce personnage de la fable cède d'autant plus au courroux qu'il découvre son reflet dans l'eau d'un ruisseau. La Fontaine compare le ruisseau aux Maximes de La Rochefoucauld auquel il dédie cette fable ${ }^{(16)}$. Le miroir ainsi que celui qui s'y mire font donc figure, chez Lizardi tout comme chez La Fontaine, de métaphore pour l'affabulation à laquelle se livre un lecteur que la fable ne doit ni épargner ni flatter.

Ce danger de la flatterie est dû, comme l'écrit Lizardi dans ses articles publiés dans les journaux, à une liberté de presse limitée. Ce faisant, il insiste sur les dangers qui pèsent sur un état où une presse censurée a pour but de flatter le gouvernement en place $^{(17)}$. En tenant compte de cet arrière-fond, les fables de Lizardi semblent donc bien être dirigées contre la flatterie et font figure de plaidoyer pour la liberté de la presse. "La Libertad de la imprenta es también el medio más eficaz para que se corrijan muchos abusos, cuya noticia no puede llegar, tal vez, por otro conducto, al conocimiento de los que gobiernan"(18)

Le fait de masquer l'amertume de certaines vérités qui pourraient toucher telle ou telle personne peut-il être aussi considéré comme une forme atténuée de la flatterie? Dans les premières lignes d'un article datant de l'année 1812, Lizardi se demande, à travers des citations de Phèdre, Térence, Juvénal et Ovide,si contrefaire la critique en optant pour une voie indirecte peut trouver sa justification. Il répond à la question par la négative et préfère le discours clair et net. Sous forme de thèse, il proclame alors : "La ignorancia ha sido el apoyo del despotismo, y la marca de los pueblos esclavizados."(19) Déjà comme journaliste, Lizardi pensait qu'il était de son devoir de patriote d'éviter toute adulation: "La adulación, repito, es una vileza, es el engaño mismo con capa de virtud, es el escalón del inepto y el apoyo del

(7) Fernández de Lizardi, El Pensador Mexicano, p. 213.

(8) Cf. Christoph Strosetzki, Die Rezeption der europäischen Antike im Lateinamerika des 19. Jahrhunderts, Stuttgart (Steiner) 1989

(9) Cf. Christoph Strosetzki, "Fénelon et Fernández de Lizardi. De l'absolutisme au libéralisme", in : Euvres et Critiques, XIV, 2, sous presse.

(10) Au sujet des fables de La Motte, Aubert, Imbert, Dorat etc. cf. : Friederike Hassauer, Die Philosophie der Fabelliere. Von der theoretischen zur praktischen Vernunft. Untersuchungen zu Funktions - und Sirukturwandel in der Fabel der französischen Aufklärung, Munich 1986.

(11) Le recueil de fables de Iriarte, qui en 1777 transcrit la poétique d'Horace en espagnol comprend principalement une illustration de la théorie classique de la poésie. Cf. : Manfred Lentzen, "Tomás de Iriartes Fabeln und der Neoklassizismus in Spanien. Ein Beitrag zur Erforschung des spanischen 18. Jahrhunderts" in : Romanische Forschungen 79, 1967, p. 603-620; R. Merritt Cox, Tomás de Iriarte, New York (Twayne) 1972; Russel P. Sebold, Tomas de Iriarte: Poeta de "Rapto Racional", Oviedo 1961 (Cuadernos de la cátedra Feijoo 11).

(12) Felix María de Samaniego, Fábulas, (E. Jareño ed.) Madrid (Castalia) 1969; Thomas Noel, Theories of the Fable in the Eighteenth Century, New York, Londres 1975 (Columbia University Press), p. 140 et suiv.

(13) Cf. Gabriel Germain, "La Fontaine et les fabulistes espagnols", in : Revue de littérature comparée, XII, avril-juin 1932, p. 312-329; Paul Mérimée, L'influence française en Espagne au dix-huitième siècle, Paris 1936.

(14) Reinhard Lüthje, Die französische Fabel im gesellschaftlichen Wandel, Rheinfelden 1985 (Schäuble, Reihe Romanistik 51), p. 19.

(15) "Es propio de este cristal y otros así, sin que ultrajen el representar la imagen conforme al original. Si alguno se viere viejo, tuerto o corcovado aquí, échese la culpa s sí y no al autor del espejo.

José Joaquín Fernandez de Lizardi, Obras I, Poesias y Fábulas, Jacobo Chencinscy (ed.), Mexico 1965 (Univ. Nacional Aut. de México), p. 290.

(16) La Fontaine, Euvres completes I. Fables, contes et nouvelles, R. Groos, J. Schiffrin (Ed.), p. 40.

(17) Fernández de Lizardi, El Pensador Mexicano, p. 35 et suiv., 39 et suiv.

(18) Fernández de Lizardi, El Pensador Mexicano, p. 42.

(19) Fernández de Lizardi, El Pensador Mexicano, p. 55. 
indigno. Así que yo que no conozco en la lisonja ni el miedo, sabed que escribiré, sin recelo de vuestras mordacidades, cuantas verdades considere útiles al fin que me he propuesto." ${ }^{(20)}$ Pour illustrer son idée, il propose l'exemple du malade dont le garde, afin de flatter le médecin, prétend que le médicament prescrit fait effet bien que ce soit le contraire. Lizardi donne lui-même l'interprétation : "El reino está enfermo, y de mucha gravedad; el gobierno es el médico de cabecera que desea la salud de este enfermo; si vosotros, escritores cobardes o aduladores, que sois los asistentes, informáis al médico contra la verdad y los gritos de vuestro corazón, no es consecuencia necesaria que el enfermo perezca a pesar de la vigilancia y sensibles deseos del diestro físico? Respondes."(21)

La première fable du Livre Premier du recueil de La Fontaine, La Cigale et la fourmi, qui se trouve chez Samaniego dans une simple paraphrase à la deuxième place, se métamorphose dans l'œuvre de Lizardi $^{(22)}$. De la cigale, l'auteur fait une tortue qui, enfermée au fond d'un puits, ne vit tout au long de l'année que de vermisseaux. Elle envie la fourmi, qui l'été peut faire des provisions pour l'hiver. Dans l'obscurité de sa prison elle n'a pas envie, comme la cigale de La Fontaine, de chanter tout l'été. Ses activités sont limitées à dormir à l'ombre et se réchauffer au soleil. La fourmi ne comprend pas que la tortue ne puisse vivre autrement et conclut en indiquant dans la fable que la misère est une punition bien méritée pour qui ne travaille pas. Remplacer la cigale par une tortue permet à Lizardi de présenter l'irresponsabilité de cette dernière, le malheur étant conditionné par les circonstances. Il s'agit donc bien ici d'une variation consciente par rapport au modèle.

Dans ses écrits journalistiques, Lizardi qualifie la suprématie coloniale espagnole de despotisme ne respectant aucune loi sinon celle de servir l'égoïsme et l'intérêt de la classe dirigeante. Sous l'égide espagnole tous les habitants ont été traités comme esclaves et les Indiens ont été considérés comme moins que des bêtes ${ }^{(23)}$. Pour lui, l'origine de la révolution, qui marqua son époque, est à chercher dans les gouvernements qui se sont jusqu'alors succédé et qui, "a los americanos se les han atado las manos para la industria, y se les han cerrado las puertas para los empleos."(24) En fait les compatriotes de Lizardi paraissent bien avoir été condamnés à une inactivité semblable à celle de la tortue de la fable. S'il y avait plus de travail, la paresse viendrait à disparaître. Elle est, pour le fabuliste, non pas la cause, mais la conséquence de la pauvreté qui règne en général au Mexique $^{(25)}$.

Très souvent les fables de Lizardi ressemblent à des variations, faites à partir de modèles. Toutefois elles sont, dans certains cas, plus étroitement liées aux fables de La Fontaine, comme, par exemple dans El gato y el ratón, où l'action ainsi que le choix des animaux ressemblent fort à ceux de la fable $L e$ Chat et le rat de La Fontaine ${ }^{(26)}$. Dans les deux cas, le chat, malgré de multiples efforts, ne parvient pas à gagner la confiance du rat. Chez Lizardi, les raisons de la méfiance du rat sont exposées longuement puisque celui-ci compte tous les membres de sa famille que le chat a déjà dévorés. Il s'exclame donc, en résumé : “¿Cómo no has de ser malo, si eres gato?" Lizardi conclut dans sa fable par le souhait de voir tous les hommes se conduire avec la même prudence avisée que celle dont fait preuve le rat. Car l'humeur du chat pourrait bien, vu sa force et sa puissance, se transformer à tout moment en brutalité.

C'est bien de cette humeur changeante et arbitraire du despote qui caractérise le pouvoir colonial que s'indigne Lizardi à juste titre. En rapport avec la suprématie coloniale, Lizardi, dans l'un de ses écrits, qualifie les despotes de "tigres racionales", qui comme "monstruos de la humanidad" sont capables de tous les crimes et ont ruiné ceux de leurs sujets qui se trouvaient dans les "cadenas de arbitrariedad"(27). Les tigres ont ici visiblement les mêmes pensées que le chat de la fable.

Alors que, dans les fables que nous avons jusqu'à présent évoquées, Lizardi emprunte ses personnages et la structure de l'action à La Fontaine, tout en donnant à celles-ci un nouveau centre de gravité, il n'en va pas de même des fables dont le nombre est beaucoup plus important et dont ce n'est que la morale que nous reconnaissons, alors que le façonnage de la fable est tout autre. Lizardi fait observer par exemple qu'il est dangereux de renier sa propre nature ou bien la situation qui vous est habituelle. Son El mono y el cazador met en garde contre une curiosité exagérée tout comme Le Rat et l'huitre de La Fontaine ${ }^{(28)}$. Dans La paloma, el cuervo y el cazador l'on voit soulignée l'idée chère à La Fontaine, de ne pas agir contre sa propre nature $^{(29)}$. Ainsi ne devrait-on pas se parer des plumes d'un autre afin de se rendre plus important. Tandis que La Fontaine expose cette idée dans Le Geai paré des plumes du paon en songeant au plagiat, Lizardi présente dans $E l$ mono vano un singe qui, richement paré et vêtu, se croit "muy diferente" puisqu'il est "hábil, rico y bien plantado"(30). Ceci

(20) Fernández de Lizardi, El Pensador Mexicano, p. 64 et suiv.

(21) Fernández de Lizardi, El Pensador Mexicano, p. 66; Samaniego par contre, qui dans La hermosa y el espejo utilise lui aussi le miroir, ne se préoccupe que de la beauté féminine dont il faut conserver la pureté. Félix María Samaniego, Fábulas : Poetas líricos del siglo XVIII, Leopoldo Augusto de Cueto, (ed.), Madrid 1929 (BAE) p. 389.

(22) La Fontaine, Fables, p. 31; Samaniego, Fábulas, p. 358; Fernández de Lizardi, Fábulas, p. 293 et suiv.

(23) Fernández de Lizardi, El Pensador Mexicano, p. 49, 50.

24) Fernández de Lizardi, El Pensador Mexicano, p. 67.

(25) Fernández de Lizardi, El Pensador Mexicano, p. 69.

(26) La Fontaine, Fables, p. 208 et suiv.; Fernández de Lizardi, Fábulas, p. 305 et suiv.

(27) Fernández de Lizardi, El Pensador Mexicano, p. 54-57.

(28) La Fontaine, Fables, p. 190 et suiv.; Fernández de Lizardi, Fábulas, p. 349-350.

(29) Fernández de Lizardi, Fábulas, p. 341 et suiv.; La Fontaine, "Le Loup devenu berger" (livre III, fable 3, p. 75 et suiv.) "Le Pot de terre es le por de fer" (livre V, fable 2, p. 117) et "La Tortue et les deux canards" (livre X, fable 2, p. 245 et suiv.).

(30) Fernández de Lizardi, Fábulas, p. 370 et suiv.; La Fontaine, Fables, p. 100. 
conduit Lizardi à écrire un véhément plaidoyer pour l'égalité des hommes entre eux :

Eres Mono, aturdido,

y Mono como todos,

aunque por raros modos

te quieras disfrazar con el vestido.

Con este desenfado,

lo misno diría yo al rico que creyó

que no es igual al probe desdichado.

De un padre descendemos;

mil pasiones sentimos;

enfermamos, morimos

todos y ser iguales no queremos

L'idée de l'égalité qui est au centre de ses travaux de journaliste semble si primordiale à Lizardi qu'il ajoute au "probe desdichado" l'explication suivante : "Esencialmente todos somos iguales, y por esta rezón nadie debería envanecerse sobre los miserables, creyéndose debería distinta que ellos"(31). Les distinctions obtenues grâce à un titre de noblesse, au talent ou à quelque mérite sont, il est vrai, légitimes, mais simplement accidentelles ${ }^{(32)}$.

Nombreuses sont aussi les similitudes entre Lizardi et La Fontaine dans l'idée qu'ils ont des relations humaines. L'inimitié, l'amitié, l'amour, ainsi que bons et mauvais conseillers, y jouent un rôle particulier ${ }^{(3.3)}$. De toute façon, le développement de la morale est chez Lizardi particulièrement détaillé et intimement lié à la situation de l'époque. La chose est évidente lorsque l'on considère la fourmi blessée qui se venge de l'éléphant par de si nombreuses piqûres dans les narines que celui-ci meurt à cause des coups qu'il se donne lui-même. La fourmi déclame en conclusion :

- A ninguno
debes agraviar de modo alguno,
y a los hombres en ti yo bien enseño
que ningún enemigo es tan pequeño
como una Hormiga coja
para tomar venganza si se enoja ${ }^{(3-1)}$.

Ici Lizardi se fait donc le défenseur du plus faible qui ne doit pas être sous-estimé par celui qui prétend être le plus fort. Dans le contexte de l'époque de la révolution mexicaine ceci se rapporte bien aux relations entre souverain et sujet. Ce sont ces mêmes rapports que La Fontaine avait masqués dans la fable Les Membres et l'estomac. Dans cette fable, La Fontaine observe, en rapport avec le souverain absolu, le fait que le profit que l'un peut tirer de l'autre a un caractère de réciprocité, sans oublier que les sujets que l'on a persuadés de cette réciprocité pourraient bien retourner heureux à leur tâche ${ }^{(35)}$. Lorsque ce thème chez Lizardi est dissimulé dans la fable du marteau et de l'enclume, celleci étant finalement sans cesse soumise aux coups du marteau - la réciprocité du profit à tirer l'un de l'autre est très peu plausible. Elle le devient davantage lorsque le marteau explique à l'enclume, qui se plaint, qu’ils sont semblables et que leur égalité vient du fait qu'ils proviennent tous deux du même matériau. Finalement le forgeron avait bien dû avoir une idée derrière la tête en créant ces objets. Donc, on doit les respecter tels qu'ils sont. Tandis que l'enclume se laisse persuader sur le champ, il n'en est pas de même pour tous et dans la dernière phrase l'auteur forme le vœu que tous les hommes puissent suivre cet exemple "conformándose todos con su suerte y adorando del cielo los decretos"(36)

Un coup d'œil jeté aux écrits du journaliste Lizardi peut donner l'explication à ce souhait. Ici il pense en effet que l'origine des discordes entre les Mexicains est due à "las distinciones de clases y condiciones en el estado llano por la desigualdad legal de las personas y las preeminencias y preferencias que han gozado unas sobre otras, las cuales nos tenían divididos en bandos y partidos y causaban una complicada rivalidad"(37). Pour lui, la "desunión funesta e impolítica" est "causa de los terribles daños que nos rodean"(38). Lizardi applique amitié et inimitié non seulement à la société mexicaine mais aussi aux rapports entre l'Espagne et l'Amérique latine. "Los naturales de España y de América somos unos por descendencia, por religión, por vasallaje y por sociedad; y así, ni vicios ni virtudes se pueden decir de los primeros que no se hallen en los segundos,"(39) affirme-t-il. Cette thèse, elle aussi, est à l'arrière-plan de la fable du marteau et de l'enclume.

Dans ce contexte où les libertés sont déclarées par la constitution ${ }^{(40)}$ mais où l'état agit en vérité de façon totalitaire, la relation entre le discours et l'action a une signification toute particulière. De fait elle est chez Lizardi tout comme déjà chez La Fontaine plusieurs fois évoquée. Contrairement à ce qui se passe chez La Fontaine, dans la fable qui met en scène les mêmes figures ${ }^{(41)}$, la guenon chez Lizardi se vante devant le chat d'être la plus habile des deux

(31) Fernández de Lizardi, Fábulas p. 371

(32) La fable de Lizardi où l'auteur met en scène la mite qui vient de naître et dont le vœu le plus cher serait que Jupiter luj offre de grandes ailes, ressemble à la fable de La Fontaine où La Grenouille qui veut se faire aussi grosse que le bæuf se gonfle elle-même. Tandis que La Fontaine critique l'étalage inconvenant de richesses et de dignités, il s'agit pour Lizardi dans un texte beaucoup plus long de s'apitoyer sur le sort de ceux qui, à l'aide de gros efforts, souhaitent quitter le milieu dont ils sont issus et font par là leur propre malheur. Cf. La Fontaine, Fables, p. 32 et suiv.; Fernández de Lizardi, Fábulas, p. 307-309.

(33) Pour La paloma celosa (p. 331 et suiv.) cf. Le Lion amoureux (livre IV, fable 1); pour La hormiga y el elefante (35355) cf. Le Lion el le moucheron (livre II, fable 9); pour Los dos lobos amigos (360 et suiv.) cf. Paroles de Socrate (livre IV, fable 17) et Le Vieillard et ses enfants (livre IV, fable 18); pour Los consejos de la rata (372 et suiv.) cf. Les Loups et les brebis (livre III, fable 13 ); pour El novillo y el toro viejo (337 et suiv.) cf. Le Chat et un vieux rat (livre III, fable 18); dans ce dernier cas la référence au taureau est bien la preuve de la tentative de l'auteur qui veut introduire dans ses fables une certaine couleur locale mexicaine.

(34) Fernández de Lizardi, Fábulas, p. 355.

(35) La Fontaine, Fables, p. 74 et suiv.

(36) Fernández de Lizardi, Fábulas, p. 352.

(37) Fernández de Lizardi, El Pensador Mexicano, p. 501

(38) Fernández de Lizardi, El Pensador Mexicano, p. 502

(39) Fernández de Lizardi, El Pensador Mexicano, p. 70.

(40) Il s'imagine dans une époque "en que por Constitución somos libres, somos ciudadanos" Fernández de Lizardi, El Pensador Mexicano, p. 58.

(41) La Fontaine, Le Singe et le chat (livre IX, fable 17); le contenu ressemble plutôt à celui de L'Enfant et le maître d'école (livre I, fable 19), où des propos inutiles ne font que retarder l'action opportune. Cf. aussi Lizardi El tigre hipócrita y el Leopardo. Fernández de Lizardi, Fábulas, p. 368 et suiv. 
pour la chasse aux souris. Hélas, lors d'une première tentative, elle se brise les os du crâne. "Murmurar cuesta poco, ¿pero hacer? Eso sí, como la Mona" $(42)$.

Si l'on prend en considération les nombreux plans et esquisses de Lizardi, qu'il avait, partisan d'une réforme, ébauchés et publiés, celui-ci est tout à fait conscient de la difficulté issue d'une transposition de ses idées dans la réalité. C'est ainsi qu'il clôt son article sur la réforme des écoles par le souhait : "Gloria y honor eterno al primero que agite por la ejecución de tan benéficos como necesarios proyectos!"(4.3)

Lizardi méprise dans la fable El loro en la tertulia les discours politiques incompétents. Dans la fable, on octroie à tort un esprit raisonnable au perroquet, tout comme au singe dans Le Singe et le dauphin de La Fontaine ${ }^{(44)}$. Lizardi fait en sorte que tous les animaux se rencontrent en une assemblée paisible et amicale. Tout de suite, le perroquet fait une très forte impression sur le public jusqu'au moment où l'on se rend compte qu'il ne sait même pas de quoi il parle : situation qui conduit Lizardi à une généralisation où il a certainement, non pas en dernier lieu, songé aux rassemblements autour des représentants du peuple :
Mas yo la fábula aplico
al tonto a quien la comprende,
que habla de todo y no entiende
lo que habla, como el Perico ${ }^{(45)}$.

Lizardi montre bien son vrai visage de réformateur libéral lorsque, non seulement il rejette tout mot superflu, mais aussi toute action précipitée. Il condamne dans El viejo y las pulgas l'activisme aveugle. Nous voyons, dans cette fable, un vieillard qui, voulant tuer de la main quelques puces gênantes, provoque de la sorte le réveil de dizaines et de dizaines d'autres puces qui ne font que rendre sa situation encore plus intolérable ${ }^{(46)}$. Telle est la leçon que nous donne la fable :

\section{Quisiera que este viejo \\ desengañado \\ enseñara paciencia \\ en los trabajos ${ }^{(4)}$}

Ici, comme déjà dans la fable La hormiga y el elefante, Lizardi utilise La Fontaine dans Le Lion et le moucheron ${ }^{(48)}$.

Dans les fables de Lizardi que nous avons citées et qui de par le thème et le motif peuvent se comparer aux fables de La Fontaine, la dimension politique est des plus évidentes. Ceci ne nous étonne guère si l'on songe que la rhétorique, dans l'œuvre journalistique de Lizardi, lui permet l'amplification de ses thèses grâce à de nombreuses métaphores et comparaisons. Maintes fables peuvent donc être lues comme servant d'exemples aux thèses présentées par le journaliste ${ }^{(49)}$.

Mais aussi dans les fables où nous n'avons pu trouver aucun rapport avec La Fontaine, l'engagement politique de Lizardi est évident. Ainsi dans El médico, la enfermedad y el paciente, le médecin se rend compte que la maladie de son patient va en s'aggravant malgré les médicaments prescrits, parce que le malade ne suit pas son régime. "Supuesto que sin régimen ni dieta, de nada sirve la mejor receta"(50). Ce qui, telle la constitution libérale, n'existe que sur le papier, mais n'est pas mis en œuvre par une volonté consciente, semble donc être condamné à l'échec. Une intelligence et un savoir raisonnable sont donc nécessaires. Ainsi Lizardi insiste-t-il donc dans le dialogue entre La espada y el sombrero sur la suprématie de la raison sur la force $^{(51)}$ et dans la fable El payo y el colegial sur la valeur du savoir et de l'éducation ${ }^{(52)}$.

Le savoir et la prise de conscience de la nécessité de l'effort et de la responsabilité vis-à-vis de soimême sont aussi des thèmes centraux dans les articles de presse de Lizardi. L'auteur ne se lasse pas d'insister sur l'importance de l'éducation. Celle-ci doit commencer aussitôt que possible ${ }^{(53)}$ et se trouve bien mal distribuée ${ }^{(54)}$. Toutefois ce ne sont pas les seigneurs coloniaux espagnols qui, à la façon de despotes orientaux, auraient maintenu les Indiens d'Amérique et les Mexicains dans leur état d'ignorance. Nous nous souvenons de la fable du médecin et de son patient, où Lizardi se plaignait du fait que, malgré la sagesse des lois et des prescriptions, l'effort venant du malade alors avait fait défaut ${ }^{(55)}$. Dès son plus jeune âge, il faudrait donc montrer à l'enfant quels sont les avantages dont jouit le niño instruido par rapport au muchacho necio ${ }^{(56)}$.

L'un des buts de toute éducation est aussi la théorie qui soutient que l'individu doit profiter à la société. Le contraste entre beauté et utilité que La Fontaine développe dans Le Cerf se voyant dans l'eau se retrouve chez Lizardi, dans le dialogue entre le chacal et le chien de manchon ${ }^{(57)}$. Ce dernier que l'on nomme aussi "Marquesito" se vante de savoir, grâce à sa beauté, rester assis, japper, danser, participer aux fêtes, jouer au soldat avec une canne oubliée. Le chacal lui rétorque qu'il est quant à lui, il est vrai, bien incapable de créer quelque distraction esthétique. Mais en contrepartie, il fait - ce qui est apprécié et reconnu de tous - "una cosa incompa-

(42) Fernández de Lizardi, Fábulas, p. 334.

43) Fernández de Lizardi Fábulas, p. 437; cf aussi : "El proyecto es de los más fáciles de practicar, de los más útiles, de los más necesarios, y cuyas benéficas consccuencias aún no se pueden calcular exactamente." Ibidem, p. 454.

(44) Fernández de Lizardi, Fábulas, p. 362-367; La Fontaine, Fables, p. 98 et suiv.

(45) Fernández de Lizardi, Fábulas, p. 366 et suiv.

(46) Fernández de Lizardi, Fábulas, p. 362 et suiv.

47) Fernández de Lizardi, Fábulas, p. 364.

(48) La Fontaine, Fables, p. 58 (2. livre, fable 9).

(49) Cf. Jürgen Grimm, La Fontaines Fabeln, Darmstadt 1976 p. 23. Pour son rapport avec l'emblème of. Barbara Tiemann, Fabel und Emblem. Gilles Corrozer und die französische Renaissance-Fabel, Munich 1974.

(50) Fernández de Lizardi, Fábulas, p. 324

51) Fernández de Lizardi, Fábulas, p. 315-317.

(52) Fernández de Lizardi, Fábulas, p. 300 et suiv.

53) Fernández de Lizardi, El Pensador Mexicano, p. 106 et suiv.

(54) Fernández de Lizardi, El Pensador Mexicano p. 419.

55) Fernández de Lizardi, El Pensador Mexicano, p. 420.

(56) Fernández de Lizardi, El Pensador Mexicano, p. 427.

57) Le Fontaine, Fables, p. 139; Fernández de Lizardi, Fábulas, p. $318-320$. 
rablemente provechosa a cualquier ciudad, reino o Estado"(58), quand il nettoie les villes en les débarrassant des viandes pourries. En ce sens, Lizardi voudrait aussi, en une ébauche de la construction d'un état idéal qu'il a un jour esquissée, accorder le droit d'être citoyen à "todo hombre que de cualquier modo le sea útil"(59).

Ce besoin d'efficacité avait déjà été exprimé dans la préface du recueil et se faisait encore plus évident dans la devise de Phèdre que Lizardi, tout comme l'avait fait Félix María Samaniego avant lui, avait placée comme épigraphe en tête de son œuvre(t(0))

Dans deux de ses fables, Lizardi réfléchit sur sa propre activité en tant que fabuliste et observateur de la société. Dans Esopo y los animales, il invite, à travers le poète Ésope, les animaux - chacun étant jaloux de l'autre, et mécontent de son propre sort à expliquer quel est le revers de la médaille, dans la destinée des animaux dont il prétend qu'ils ont plus de chance que lui :

\section{Todos callaron. Ninguno \\ desde entonces solicita \\ trocar con otros su suerte, \\ y contentos hasta el día \\ con la suya, viven libres \\ de tenores y de envidias.}

Así el hombre viviera

si la suerte que invidia conociera ${ }^{(61)}$.

Ici, c'est Ésope qui semble être apaisant et conciliateur. Il est certain que Lizardi par le fait des contradictions dans la politique intérieure et de la guerre d'indépendance a voulu jouer lui-même un rôle conciliateur, dans lequel - tout comme Florian avec ses fables lors de la révolution française ${ }^{(62)}$ - il se serait efforcé d'éviter les conflits superflus. Le fait qu'il ait eu l'intention d'intervenir est évident lorsque l'on examine la fable qui clôt le recueil. Dans $E l$ palacio de naipes nous voyons deux enfants construire avec effort un château de cartes. Tout à coup survient un courant d'air qui détruit en un instant la forteresse. Le père console son enfant en lui expliquant qu'il ne vaut pas la peine de se plaindre de la perte d'une "obra sin substancia".

De nada sirve, pues que la obra esté hecha,

si lánguida o inútil no aprovecha.

Cuando yo leo mis fábulas despacio temo corran la suerte del palacio ${ }^{(6.3)}$

Il en est de même des fables de Lizardi. Elles seraient inutiles si elles ne servaient à personne. Et c'est bien sûr le contraire que souhaite Lizardi car il n'aurait pas à craindre alors que ses fables ne partagent le sort du château de cartes.

En résumé, nous pouvons donc retenir que les fables de Lizardi portent souvent l'empreinte de la constellation des figures et des leçons contenues dans les fables de La Fontaine. Ceci ne nous étonne nullement si nous nous référons à la présence culturelle française dans le Mexique du XIX ${ }^{c}$ siècle et à la connaissance profonde que Lizardi avait de la littérature française au XVII ${ }^{\mathrm{c}}$ siècle.
Ses fables nous semblent bien porter la marque d'une prudence nécessaire au journaliste de son époque. Dans celles-ci, Lizardi ne choisit pas, par opposition à la limitation de la liberté de la presse et à la censure, le chemin facile de la flatterie, mais veut offrir à la réalité contemporaine le reflet de ce qu'elle est. Lizardi préfère donc formuler un jugement honnête sur son époque. Il lui faut par conséquent faire d'abord son propre examen de conscience et se demander dans quelle mesure il doit masquer, à travers la fable, la vérité sous une forme extérieure peut-être trop complaisante, courant ainsi le risque que l'efficacité de l'énoncé ne passe quasiment inaperçu, cédant ainsi le pas aux éléments distrayants qui sont au cœur de toute fable.

Toutefois, nous avons pu constater que les fables de Lizardi exposent bien les idées de son manifeste politique, surtout si l'on compare les fables aux écrits journalistiques de l'auteur. Ainsi la tortue se plaint-elle du fond du puits de l'inactivité à laquelle la puissance coloniale a condamné les Mexicains. C'est cette puissance qui est symbolisée, à cause de l'arbitraire de son despotisme, par le chat ou bien le tigre. Le singe paré de bijoux plaide pour l'abolition, dans la société, de privilèges injustifiés, et l'enclume pour la suppression de l'antinomie existant entre colonisés et colonisateurs. Le fait que l'action réfléchie du faible aboutisse à tout, et que l'action irréfléchie du puissant n'aboutisse à rien, est décrit à travers la fourmi auprès de l'éléphant ou bien à travers le vieillard qui chasse ses puces. Les faibles ne doivent pas être sous-estimés, nous démontre la fourmi aux prises avec l'éléphant. Et la meilleure des constitutions ne reste qu'une théorie pure et simple si elle n'est ni porteuse d'idées raisonnables, ni animée d'actes sincères; c'est ce que nous prouvent la guenon face au chat, le malade face au médecin, l'enfant inculte face à celui qui a reçu une bonne éducation.

De toute façon, il est évident que, pour Lizardi, les fables sont un instrument mis au service du message politique. Leur utilité didactique est une force incontestable que le poète libéral situe au-dessus de leur valeur esthétique et divertissante. Et voilà bien la distinction primordiale que nous pouvons faire entre La Fontaine et Lizardi.

Christoph STROSETZKI Université de Cologne

(58) Fernández de Lizardi, Fábulas, p. 320.

(59) Fernández de Lizardi, El Pensador Mexicano, Augustín Yáñez (ed.), Mexico 1954, p. 147.

(60) "Duplex libelli dos est : quod risum movet, es quo prudenti, vitam, consilio monet" (Fedro, Fábulas, Prólogo, libro I), Fernández de Lizardi, Fábulas, p. 285; Samaniego, Fábulas, in Leopoldo Augusto de Cueto (ed.), Poetas líricos del siglo XVIII, Madrid 1929, p. 356.

(61) Fernández de Lizardi, Fábulas, p. 299.

(62) Cf. Hinrich Hudde : "Regression angesichts der Revolution", in Zeitschrift für französische Sprache und Literatur 87,1977 , p. 99-141; pour La Fontaine dans ce contexte cf. Jürgen Grimm, "Grande est la gloire ainsi que la tuerie". Guerre et rhétorique dans les Fables de La Fontaine, in Cahiers d'histoire des Littératures Romanes, 1989 1/2, p. 162178 .

(63) Fernández de Lizardi, Fábulas, p. 375. 
Discussion de la communication de M. STROSETZKI.

M. Barchilon demande au conférencier s'il a rencontré des témoignages explicites sur le danger représenté par la portée critique des fables de Lizardi aux yeux des autorités mexicaines de son temps.

M. Strosetzki répond qu'après avoir été emprisonné pour s'être exprimé ouvertement dans la presse, Lizardi a contourné la censure par le recours à l'écriture littéraire, notamment par ses Fables, qui paraissent avoir échappé à la vigilance des censeurs. Il n'écrivait au demeurant que pour la minorité éclairée qui pouvait lire ses œuvres. Le nom de Lizardi jouit actuellement d'une grande notoriété au Mexique, mais plus pour ses romans que pour ses fables.

Propos transcrits par Mme Prudhomme et résumés par M. Dandrey. 